DIVERSIDAD DE HONGOS FORMADORES DE MICORRIZAS ARBUSCULARES (HMA) EN TRES AGROECOSISTEMAS CON PASTO COLOSOANA (Bothriochloa pertusa (L) A. Camus) EN EL DEPARTAMENTO DE SUCRE

\title{
DIVERSITY OF ARBUSCULAR MYCORRHIZAL FUNGI (AMF) IN THREE AGROECOSYSTEMS WITH GRASS COLOSOANA (Bothriochloa pertusa (L) A. Camus) IN THE DEPARTMENT OF SUCRE
}

FREDIS ESPITIA D, ${ }^{1}$ M.Sc, ALEXANDER PEREZ C, ${ }^{2 *}$ Dr.

${ }^{1}$ FED, Colombia.

2Universidad de Sucre, Grupo Bioprospección Agropecuaria, Colombia.

\section{Key words:}

Microorganisms, abundance,

soil,

pasture.

\section{Abstract}

The present study evaluated the diversity of arbuscular mycorrhizal fungi (AMF) associated soils from three ecosystems grown with pasture colosoana in three localities of the Department of Sucre, Colombia. Soil samples were collected, AMF spores isolated, count of spore was made and morphotypes isolated were classification at the level of species or genera. We found that the genus of AMF over prevailing in native and compacted soils in the three selected municipalities is the genus Glomus, followed by other genera to a lesser extent. In general the soils that presented greater wealth of genres of HMA were those of the municipality of San Marcos, while the lowest richness was found in the municipality of Sincelejo.

\section{Palabras Clave:}

Microorganismos, abundancia, suelo, pastura.

\section{Resumen}

El presente estudio evaluó la diversidad de hongos formadores de micorrizas arbusculares (HMA) asociadas a suelos de tres ecosistemas cultivados con pasto colosoana en tres localidades del departamento de Sucre, Colombia. Se recolectaron muestras de suelo, se aislaron esporas de HMA, se realizó conteo de espora y se identificaron morfotipos a nivel de especie y/o géneros. Se encontró que el género de HMA que más predomina en los suelos compactados y nativos de los tres municipios seleccionados es el género Glomus, seguido de otros géneros en menor proporción. En general los suelos que presentaron mayor riqueza de géneros de HMA fueron los del municipio de San Marcos, mientras que la menor riqueza se encontró en el municipio de Sincelejo. 


\section{Introducción}

Los organismos del suelo son ampliamente diversos y contribuyen a un amplio rango de servicios ambientales que son esenciales en el mantenimiento de la calidad de suelos y la función de sostenibilidad de los ecosistemas naturales y controlados (BARRIOS, 2007). Entre estos se encuentran los hongos formadores de micorrizas arbusculares (HMA), los cuales establecen una simbiosis con las raíces de las plantas desempeñando un papel clave en el reciclaje de nutrientes del ecosistema y la protección vegetal ante el estrés, la eliminación de patógenos y la descomposición de residuos sólidos, lo que incrementa el enraizamiento de las plantas, gracias a la producción de fitohormonas, enzimas y otras sustancias fitoactivas (KASCHUK et al., 2010); así como posibles bioindicadores para monitorear la calidad de los suelos (SCHLOTER, 2003; BENDING, 2004; WINDINGA, 2004; SANTANNA, 2009; SÁNCHEZ, 1999).

El establecimiento de la simbiosis va a depender de las interacciones entre los tres componente del sistema: el hongo, la planta y las condiciones ambientales. Su presencia implica que ocurran procesos de reconocimiento entre los simbiontes, compatibilidad y especificidad, los cuales condicionan su expresión y conducen a la integración morfológica y funcional de las asociaciones (GONZÁLEZ, 1996). La esporulación de HMA bajo condiciones de campo está determinada por: las condiciones físico-químicas del suelo $(\mathrm{pH}$, respiración microbiana, contenido de fósforo, temperatura, aireación, textura y contenido de materia orgánica), condiciones climáticas (intensidad y duración de la luz, humedad, épocas de lluvias y épocas secas) y por las prácticas agronómicas (preparación del terreno, aplicación de pesticidas y prácticas culturales) (AGUILERA, 2005).

La mayoría de suelos del Caribe colombiano han presentado durante las últimas décadas un intensivo empobrecimiento debido a la tala indiscriminada, el envenenamiento por uso de fertilizantes y pesticidas y el sobrepastoreo de ganado (DANE, 2010). Cabe resaltar que a la ganadería, principal actividad económica del departamento de Sucre, se le dedica un 84,9\% de los suelos exclusivamente para pastoreo, y cuya fuente exclusiva de alimento animal la constituye las leguminosas nativas y las gramíneas (UNIVERSIDAD DE SUCRE, 1996). Dentro de estas últimas, el pasto colosoana (Bothrichloa pertusa (L) A. Camus) es la especie predominante en la región, donde reporta un área extensa sembrada (aproximadamente 274.005 has); convirtiéndose en la gramínea de mayor importancia económica establecida en estos suelos como alternativa de alimentación (CHAMORRO, 1994; SIEVERDING, 1983).
Durante las últimas décadas se ha intensificado en el mundo la producción agrícola para satisfacer la demanda continua de alimentos lo que ha generado el uso intensivo y hasta desmedido de los recursos naturales. Esto ha llevado al empleo inadecuado en el manejo de los suelos lo que ha provocado su degradación y el deterioro de su calidad en vastas regiones del planeta. En Colombia, país reconocido por su gran riqueza y biodiversidad natural, también se ha manifestado este proceso de degradación de los suelos, especialmente como consecuencia de la implementación de la agricultura continua, el pastoreo intensivo y el mal manejo de prácticas agrícolas a través del uso de fertilizantes y pesticidas. Dichas prácticas provocan el deterioro de las propiedades físicas, químicas y biológicas de los suelos y el aumento de la superficie afectada por procesos erosivos y de degradación en grandes extensiones del departamento de Sucre (PEREZ et al., 2015).

Sin embargo, hasta la fecha es poco lo que se conoce sobre la actividad microbiológica en estos suelos y además no se ha realizado un estudio significativo para evaluar en forma integral la presencia de HMA como componentes biológicos del suelo y su posible rol como uno de los indicadores que determinen la calidad de los suelos en el departamento de Sucre. Por lo anterior el objetivo del presente trabajo consistió en evaluar la diversidad de hongos formadores de micorrizas arbusculares asociados al pasto $B$. pertusa (L) A. Camus, en tres agroecosistemas de las subregiones Golfo de Morrosquillo, San Jorge y Sabanas en el departamento de Sucre, Colombia.

\section{Materiales y métodos}

Tipo y sitio de estudio: El presente estudio se realizó en fincas sembradas únicamente con el pasto colosoana dedicadas a la ganadería y en zonas de bosque no intervenidas ubicadas en los municipios de Tolú, San Marcos y Sincelejo en el departamento de Sucre.

Población y selección de la muestra: La población estuvo conformada por las fincas ganaderas sembradas con pasto colosoana con pastoreo intensivo y zonas de bosque nativo no intervenidas en los municipios de Tolú, San Marcos y Sincelejo, Departamento de Sucre, Colombia.

Las muestras fueron colectadas en el primer semestre del año 2013, durante la temporada de lluvias, en dos zonas agroforestales, previamente identificadas (bosque nativo no intervenido y zona degradada o compactada) establecidas únicamente con el pasto colosoana o kikuyina (B. pertusa (L) A. Camus). En las zonas se realizó un muestreo aleatorio en forma 
de zig-zag, tomando 20 muestras de suelo a una profundidad de 0,15 y $25 \mathrm{~cm}$, respectivamente. Las muestras se depositaron en bolsas plásticas rotuladas con el nombre de la zona y fecha de recolección. Las muestras tomadas de cada zona agroforestal fueron conservadas y transportada al laboratorio de investigaciones microbiológicas de la Universidad de Sucre para el análisis microbiológico respectivo y físico químico, respectivo.

Procesamiento de muestras y aislamiento de esporas: Las muestras de suelos fueron tamizadas para separar las partes gruesas de suelos (piedras, cascajos) y raíces. Una vez tamizadas se procedió al análisis físico-químico y al aislamiento de esporas de hongos formadores de micorrizas arbusculares, mediante técnica propuesta por SIEVERDING (1983); DANIELS y SKIPPER (1991) y BOTERO, (1998).

Con la ayuda de una micropipeta se extrajeron las esporas de los diferente morfotipos encontrados, se colocaron en tubos de ensayos con agua estéril, se rotularon con el nombre de la zona donde fueron aislados y se guardaron en nevera a $4^{\circ} \mathrm{C}$ durante 3 días, para su posterior identificación. Los morfotipos aislados se depositaron en cajas de petri, se observaron al estereoscopio para detallar sus características en agua, verificar y eliminar esporas de otros morfotipos y partículas contaminantes. Una vez limpias las esporas y verificado el morfotipo, se realizó la identificaron a nivel de géneros, mediante técnicas propuestas por SCHENCK y PÉREZ (1990), MORTON (1996), OEHL et al. (2011), INVAM (2002).

\section{Resultados y discusión}

Análisis físico-químico de los suelos analizados. Un total de 20 fincas ganaderas y 20 zonas de bosques nativos con suelos moderamente intervenidos fueron muestreadas en los municipios de Santiago de Tolú, Sincelejo, y San Marcos. De acuerdo a los resultados de los análisis físico-químicos de los suelos compactados y de bosques nativos, se encontró que presentan los siguientes valores (Tabla 1).

En el municipio de Santiago de Tolú, Subregión Golfo de Morrosquillo, se muestrearon fincas ganaderas establecidas con pasto colosoana, cuyos suelos presentaban alto grado de compactación, pisoteo intensivo e inadecuadas prácticas agrícolas, y también suelos de bosques nativos que no presentaban alteración. Estos suelos están constituidos por sedimentos aluviales, marinos o combinaciones de los dos. En algunos sectores hay sedimentos orgánicos.La actividad ganadera está concentrada en un relieve de llanura fluviomarina, caracterizada por ser extensiones planas con desniveles minúsculos y pendientes suaves
Tabla 1. Análisis físico-químico de suelo en tres localidades del departamento de Sucre.

\begin{tabular}{|c|c|c|c|}
\hline Parámetro & Tolú & Sincelejo & San Marcos \\
\hline $\mathrm{pH}$ & $\begin{array}{l}\text { Ligeramente } \\
\quad \text { acido }\end{array}$ & $\begin{array}{l}\text { Ligeramente Acido a } \\
\text { moderadamente alcalino }\end{array}$ & $\begin{array}{l}\text { Fuertemente acido- } \\
\text { medianamente acido }\end{array}$ \\
\hline MO & $\begin{array}{l}\text { Normal- } \\
\text { Mediano }\end{array}$ & Bajo-Medio & Bajo - muy bajo \\
\hline$P$ & Alto- Muy Alto & Bajo - Medio & Muy bajo \\
\hline K & Alto & Medio -Alto & Moderado \\
\hline $\mathrm{Ca}$ & Medio - Alto & Medio -Alto & Muy bajo \\
\hline $\mathrm{Mg}$ & Medio - Alto & Medio -Alto & Bajo \\
\hline $\mathrm{Na}$ & $\begin{array}{l}\text { Muy Alto- } \\
\text { Excesivo }\end{array}$ & Alto & Alto \\
\hline Textura & $\begin{array}{l}\text { Franco Arenosa- } \\
\text { Franco Arcillosa }\end{array}$ & Arcillosa & Franco Arenoso \\
\hline Porosidad & Muy Satisfactoria & Muy baja & $\begin{array}{l}\text { Muy Baja - } \\
\text { Satisfactoria }\end{array}$ \\
\hline $\mathrm{ClC}$ & Baja- Media & Medio & Bajo - muy Bajo \\
\hline $\begin{array}{l}\text { Densidad } \\
\text { aparente }\end{array}$ & Media & Media & Alto - Moderado \\
\hline $\begin{array}{l}\text { Densidad } \\
\text { real }\end{array}$ & Media & Baja & adecuada \\
\hline Humedad & Media & Bajo & Bajo - Muy Bajo \\
\hline
\end{tabular}

y relieve de pequeños valles que corresponden a áreas estrechas y alargadas.

A los suelos de las fincas ubicadas en los municipios de Sincelejo y Corozal, subregión Sabana, no se les ha ejecutado ninguna práctica agrícola para mejorar sus propiedades fisicoquímicas en los últimos diez años, principalmente su aireación, ni se realiza abonamiento. Como consecuencia de lo anterior estos suelos presentan altos grados de compactación y degradación en forma general. La especie de pasto que más predomina en estos suelos es el pasto colosoana más conocida como kikuyo.

De otro parte, en el municipio de San Marcos, Subregión San Jorge, los suelos de bosques nativos muestreados no han sido intervenidos o explotados con fines agrícolas y ganaderos. Con respecto a los suelos compactados seleccionados, estos han sido objeto de explotación ganadera intensiva, sin la implementación de prácticas agrícolas que conlleven a mejorar sus propiedades fisicoquímicas, razón por la cual estos presentan altos grados de compactación. Para la ganadería bovina en la región se utiliza como fuente de alimentos pastos naturales y mejorados, encontrándose en la pastura natural especies como gramalote, canutillo, lambe, gallina de punta y colosoana.

Densidad de esporas de HMA. Las mayores densidades de esporas fueron aisladas en el municipio de Tolú para suelos compactados con respecto a Sincelejo y San Marcos (Fig. 1). Asimismo, de acuerdo 
a la prueba de Tukey, se observaron mayores valores de densidad de HMA en suelos no compactados para el municipio de Tolú con respecto a los otros suelos analizados de los municipios.

El análisis de varianza de densidad de espora realizado para suelos compactados se encontró diferencia significativa entre los suelos de los municipios evaluados. De acuerdo a la prueba de Tukey, existe diferencia significativa entre los suelos de Tolú y Sincelejo comparados con los de San Marcos, mientras que no existe diferencia significativa entre los valores registrados de los suelos de los municipios de Sincelejo y Tolú.

En los suelos compactados del municipio de San Marcos se registraron bajos valores de esporulación debido a que estos suelos presentan un valor alto de acidez (fuertemente acido), mientras que estos valores son similares (ligeramente acido) entre los suelos de los municipios de Tolú y Sincelejo. Otro indicio importante para sustentar lo anterior resultados son los valores muy bajos de fosforo en los suelos de San Marcos en comparación a los valores en los suelos de los otros municipios, debido a que la disponibilidad de este mineral está relacionado con la esporulación de micorrizas.

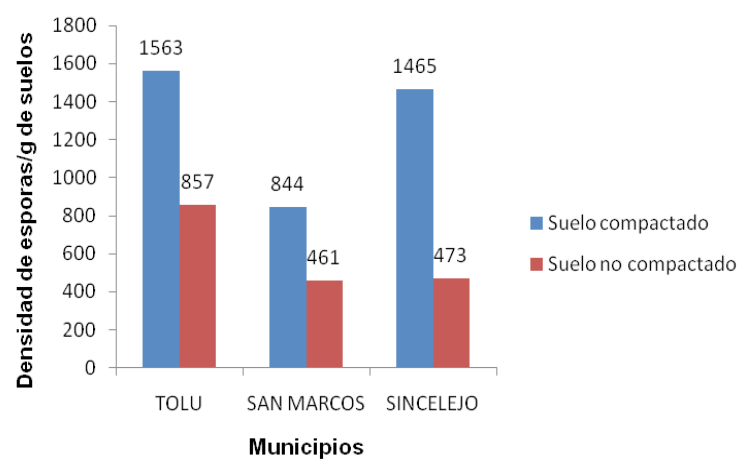

Figura 1. Densidad de esporas/ $100 \mathrm{~g}$ de suelo de HMA aisladas pasto colosoana de fincas ganaderas con respecto a municipios y tipo de suelos analizados en el primer semestre de 2013. Prueba múltiple de rangos

Los resultados encontrados en el presente estudio se compararon con el trabajo realizado por PICONE (2000), sobre diversidad y abundancia de esporas de HMA en bosques y pastos tropicales. Estos autores encontraron que el número de esporas de HMA es igual o mayor en suelo de fincas establecidas con pasturas que en suelos de bosques. El número de esporas por $100 / \mathrm{g}$ de suelos en suelo de bosques osciló en un rango de $110 \pm 770$ esporas, con respecto al de fincas establecidos con pasturas el cual presentó rangos de $830 \pm 2600$ esporas, coincidiendo estos resultados por lo expuesto por PICONE (2000). Todo lo antes expuesto ratifica los resultados encontrados en el presente estudio donde se obtuvieron las mayores densidades de esposas en suelos provenientes de fincas ganaderas con manejo extensivo desde hace aproximadamente 15 años, con respecto a los suelos de bosques.

Para el presente estudio, es muy probable que los bajos valores de densidad de esporas encontrados con respecto al trabajo realizado por PICONE (2000), en los suelos compactados sembrados con pasto colosoana, estén asociados al pastoreo intensivo a que ha sido sometido estos suelos, los cuales en los últimos años, no han tenido una manejo adecuado para mejorar sus propiedades fisicoquímicas, principalmente su aireación, ni se ha realizado abonamiento para suplir la extracción de nutrientes que la especie de pasto ha venido ejecutando a través del tiempo, lo cual es similar a lo planteado por MECINAS et al. (1991), quienes sugieren que una alta esporulación de HMA es una respuesta de los hongos a condiciones climáticas adversas. Las comunidades de HMA son dinámicas en el tiempo, y pueden ser moduladas por características del suelo (físicas, químicas y biológicas) y por aspectos climáticos, que en el caso del trópico Colombiano están asociados a la altitud (PÉREZ, 2010).

Diversidad de morfotipos de HMA en las zonas estudiadas. Para los suelos compactados y no compactados del municipio de Tolú se aislaron los siguientes morfoespecies de HMA, como se observa a continuación en la Fig. 2. Se identificaron 12 morfoespecies con características microscópicas similares (cf) a especies según claves taxonómicas determinadas por OEHL et al. (2011), OEHL y SIEVERDING (2011); GOTO (2008); SIEVERDING (2006); INVAM (2013); PEÑA (2006). En la Fig. 3 , se observa la distribución de morfoespecies de HMA, correspondiendo el máximo porcentaje para el género Glomus y el menor porcentaje para el género Acaulospora.

Un total de 31 morfoespecies (Fig. 4 a y b), fueron aislados e identificados con características microscópicas similares (cf) a especies según claves taxonómicas determinadas por OEHL et al. (2011), OEHL y SIEVERDING (2011), GOTO, (2008), SIEVERDING (2006) INVAM (2013), PEÑA (2006), asociadas a suelos compactados y no compactados asociados a pasto colosoana del municipio de San Marcos. 


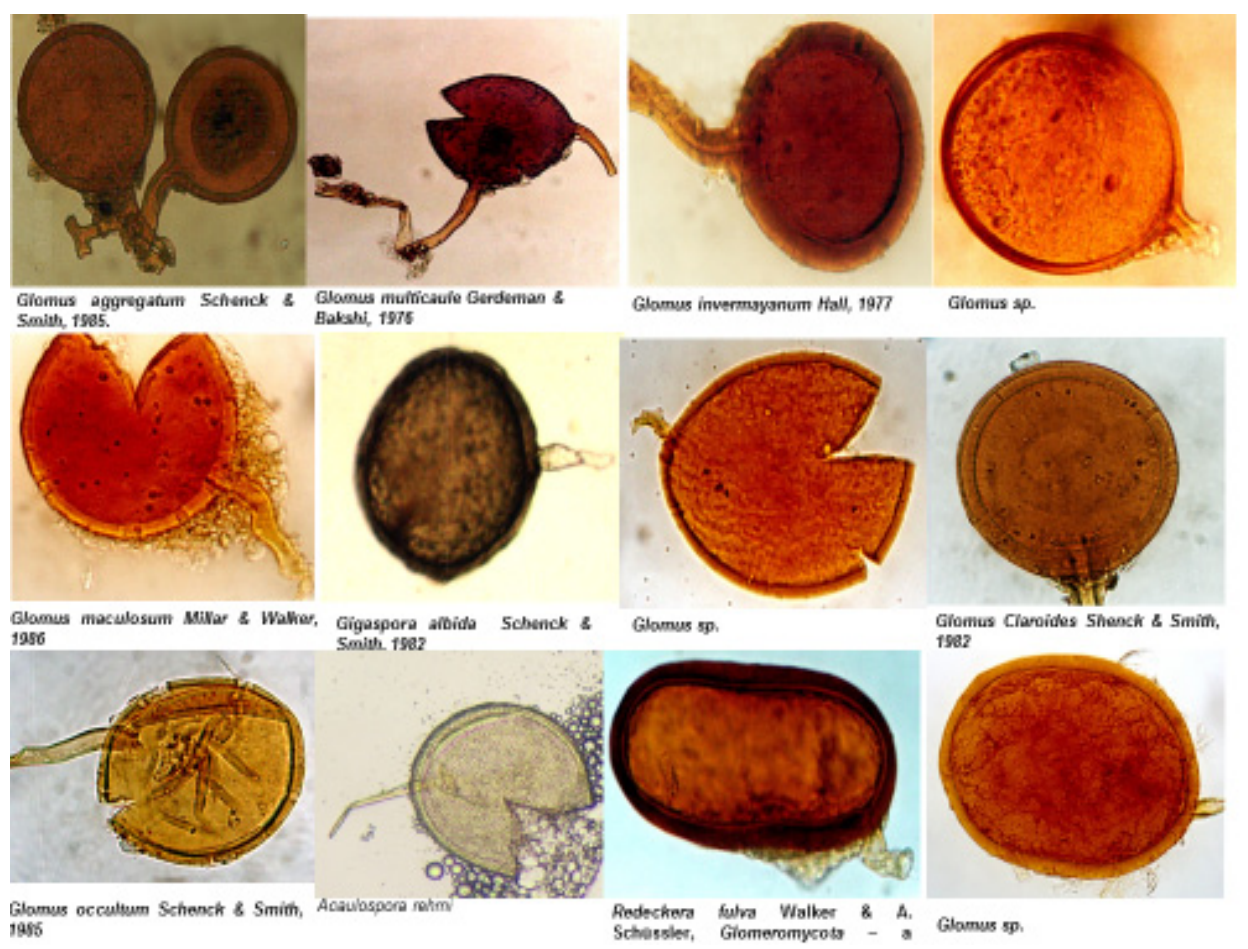

Figura 2. Diversidad de morfoespecies de micorrizas arbusculares aisladas de suelos compactados y no compactados pertenecientes al municipio de Tolú, departamento de Sucre, Colombia. Año 2013. Fuente. Espitia-Doria, 2013.

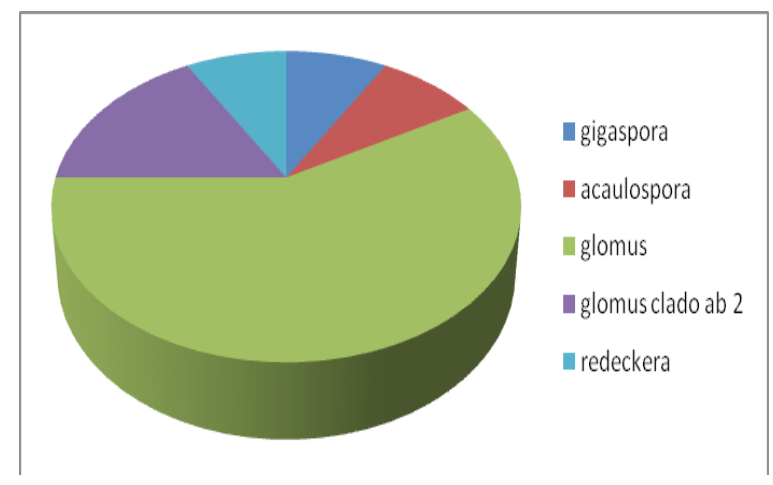

Figura 3. Porcentaje de Géneros de HMA, aislados en suelos del municipio de Tolú, departamento de Sucre, Colombia. Año 2013.

Las 31 morfoespecies aisladas fueron clasificadas taxonómicamente en 11 posible géneros: Claroideo, Glomus, Viscospora, Glomus, Ambispora, Gigaspora, Funneliformis, Intraspora, Tricispora, Acaulospora, Diversispora, SimiGlomus, de los cuales el $48 \%$ corresponde al género Glomus, seguido de los géneros Claroideo, Glomus y Diversispora con un $10 \%$, Viscospora y Gigaspora con $7 \%$ y encontrándose con un valor del $3 \%$ morfotipos, que corresponde a los géneros Ambispora, Funneliformis, Intraspora,
Tricispora, Acaulospora, SimiGlomus (Fig. 5). Los resultados anteriores concuerdan con lo reportado por ESPITIA et al. (2003), quienes en una investigación en fincas ganaderas del municipio de Corozal, Sucre, caracterizaron hongos formadores de micorrizas arbusculares (HMA) en rizósfera del pasto colosoana, donde fueron aislaron 31 morfotipos de HMA, con un porcentaje de $96,9 \%$ que correspondían a morfotipos con características similares a especie dentro del género Glomus y $3.1 \%$ al género Gigaspora.

Para los suelos compactados y no compactados del municipio de Sincelejo se identificaron 10 morfoespecies con características microscópicas similares (cf) a especies según claves taxonómicas determinadas por OEHL et al. (2011), OEHI y SIEVERDING (2011), GOTO (2008), SIEVERDING (2006), INVAM (2013), PEÑA (2006) (Fig. 6).

Las 9 morfoespecies fueron clasificadas taxonómicamente en cuatro posible géneros: Glomus Clado ab, Glomus Clado ab1, Glomus y SimiGlomus, de los cuales el $70 \%$ corresponde al género Glomus, seguido de los géneros Glomus clado ab y clado ab1 con un $10 \%$ cada uno, y el género SimiGlomus con un $10 \%$ (Fig.7). Resultados similares reportaron ESPITIA y MARTÍNEZ (2003) en estudios de HMA asociados 


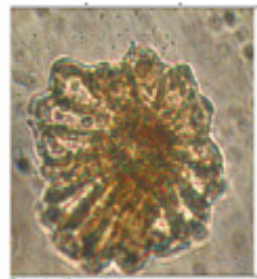

Glomes sp

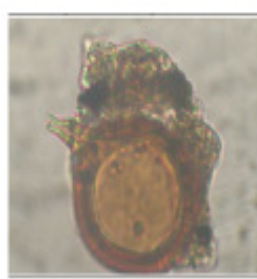

Vacospon $\$$

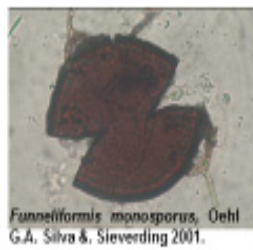

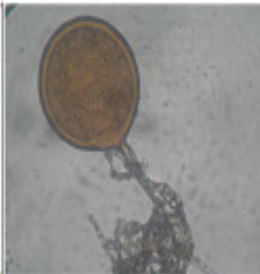

Simiglamus bai Berch \& Trappe, 995, G.A. Silva Oellin \& showerding.

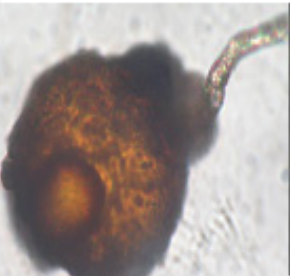

Gigsspors albido Schenck \& Sm GS 192 .

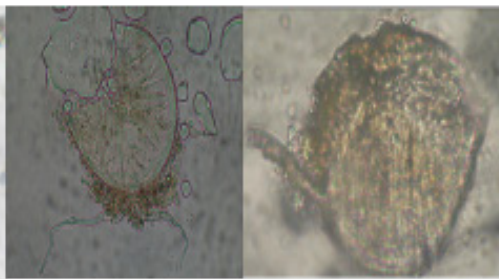

Otrensipors sp
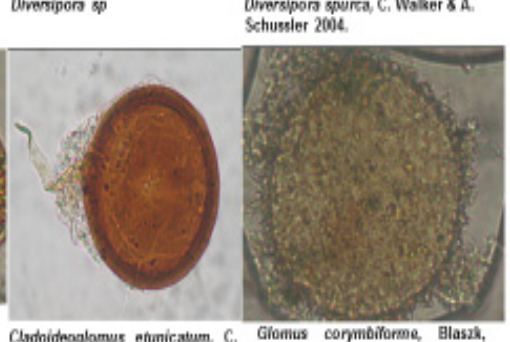
Walker \& A. Schussler 2010.

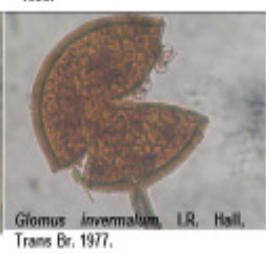

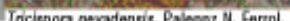

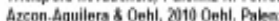
GA Silva \& Sheverd coen now.

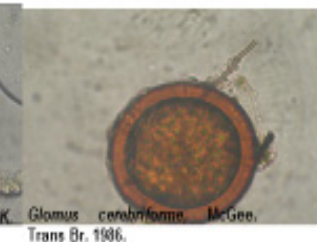

Figura 4a. Diversidad de morfoespecies de micorrizas arbusculares aisladas de suelos compactados y no compactados pertenecientes al municipio de San Marcos, departamento de Sucre, Colombia. Año2013.
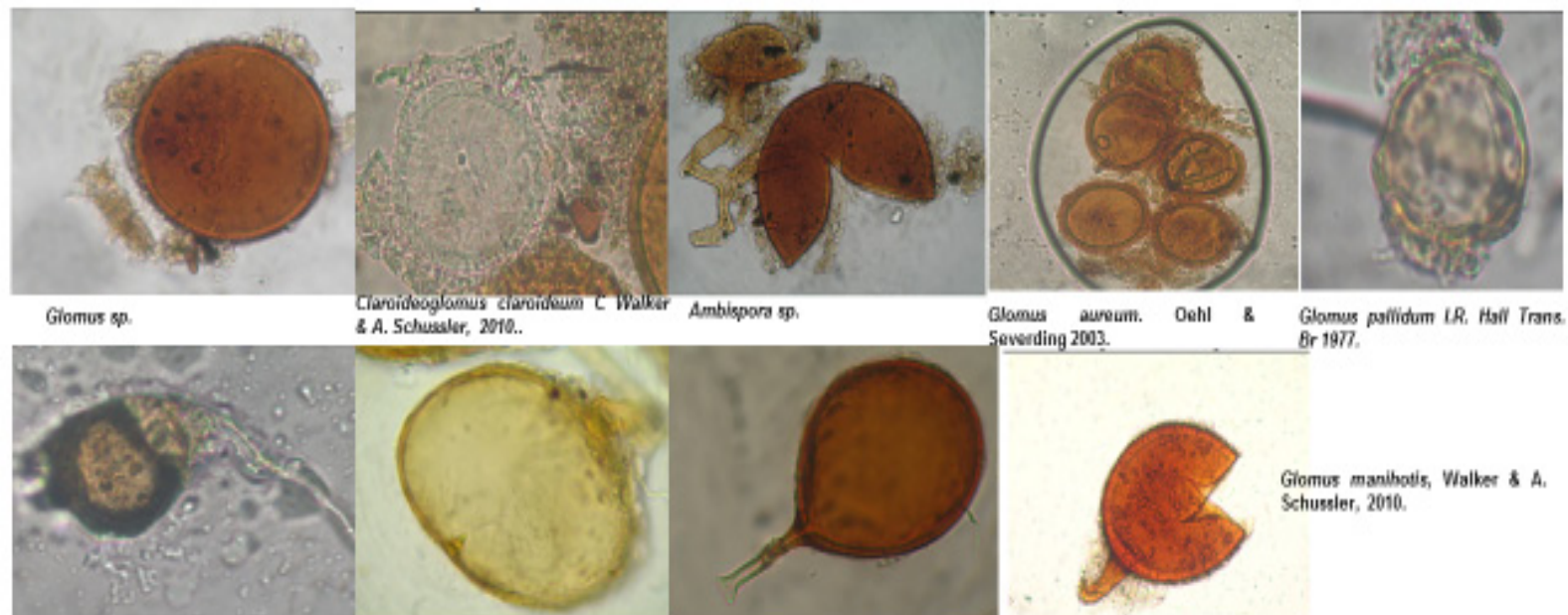

S A. Schusder, 2010.

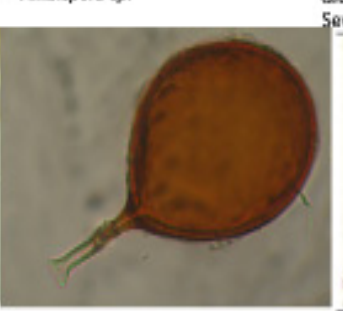
Sewerding 2003 . Br 1977 .

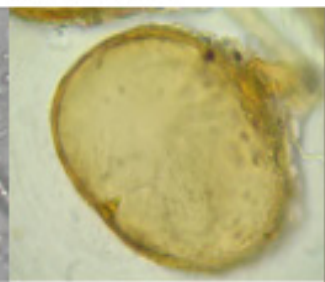

Oiversispora ebémsa, Walker \& A Chussler, 2010
Glomos ambisporum, G.S. Sm. \& NC. Chenck, 1965,

Viscospora viscosa
\& G.A.Silva, 2011.

Figura 4b. Continuación diversidad de morfoespecie de micorrizas arbusculares aisladas de suelos compactados y no compactados pertenecientes al municipio de San Marcos, departamento de Sucre, Colombia. Año 2013. Fuente: Jiménez Roberto y Ader Núñez, 2014. 


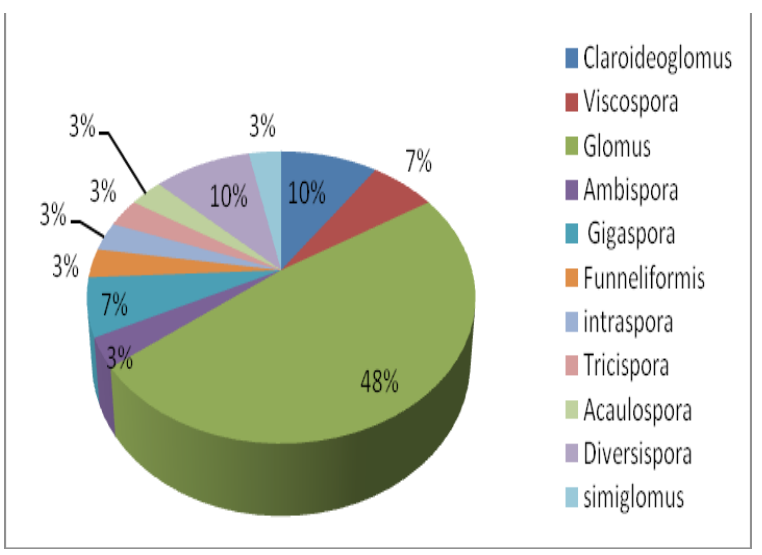

Figura 5. Porcentaje de Géneros de HMA, aislados en suelos del municipio de San Marcos, departamento de Sucre, Colombia. Año 2013. al pasto colosoana, donde aislaron 31 morfotipos de HMA, con un porcentaje de $96.9 \%$ que correspondían a morfotipos con características similares a especie dentro del género Glomus.

De acuerdo al análisis estadístico, se determinó que el género de HMA que más predomina en los suelos compactados y nativos de los tres municipios seleccionados es el género Glomus, seguido de otros géneros en menor proporción. Los suelos que presentan mayor riqueza de HMA en relación a géneros son los de San Marcos, mientras que los que menos riqueza presentan son los de Sincelejo (Fig.8). La composición micorrízica de los suelos bajo diferentes coberturas es diferente dado que la vegetación, como hospedero obligado de las micorrizas arbusculares, tiene un efecto directo sobre la diversidad de sus poblaciones (SCHENCK et al., 1989; PEÑA-VENEGAS, 2001).
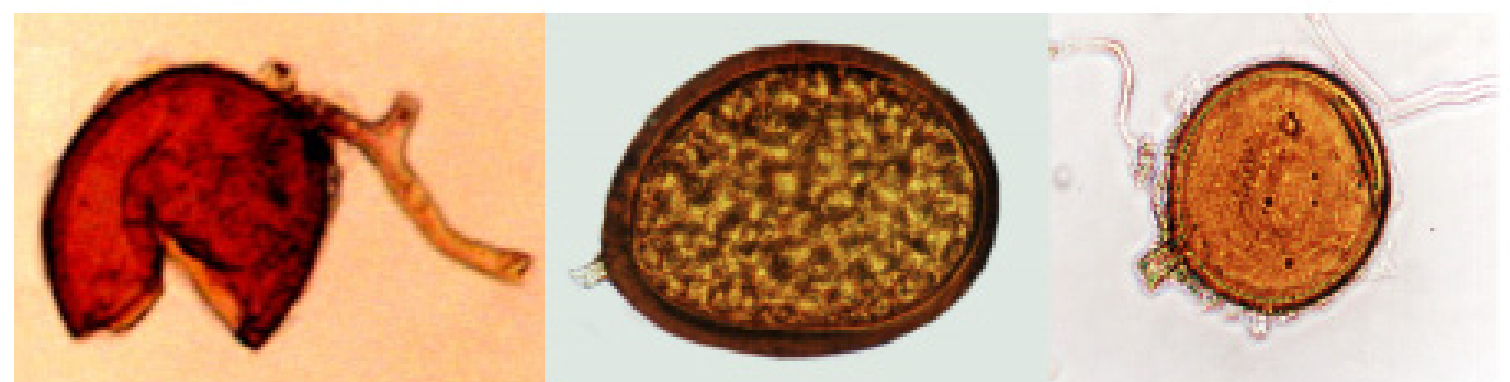

Glomus aggragatum Schenck \& Smith, Koshe, 1995 Glomus imemaium LR. Hall, Tranc. Br. 1977.

Glomus hoi Berch, S. M and Trape L. M 1995
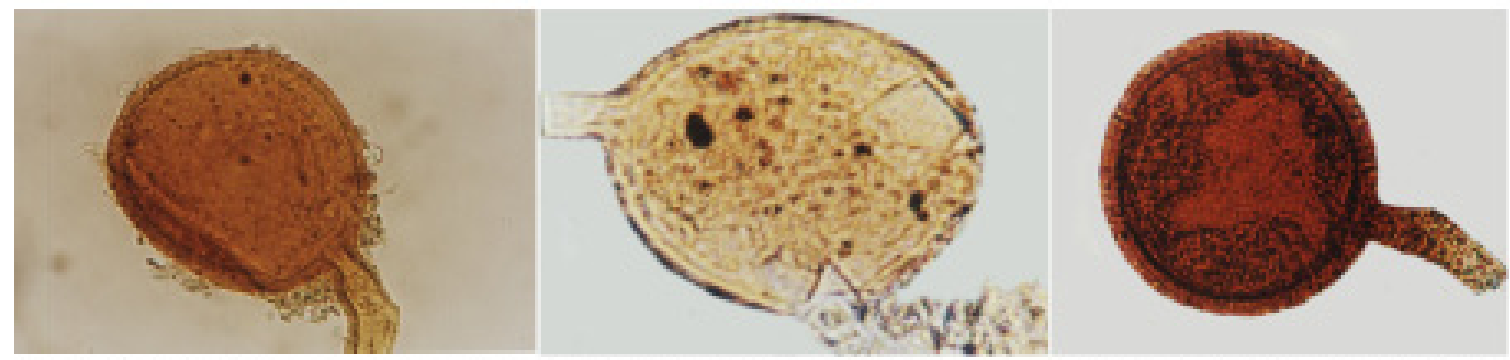

Giomus mucrocarpum Tulssne \& Tolasns, 1953

Giomus leptovichum Schenck \& Smith, 1902

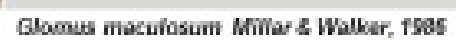

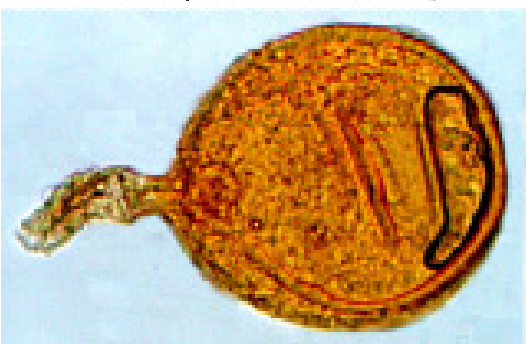

Glomus claroides Schenck \& Sminh, 1952

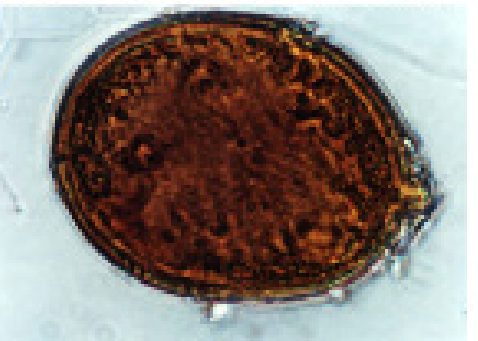

Giomus citricola Tang \& Zang, 1984

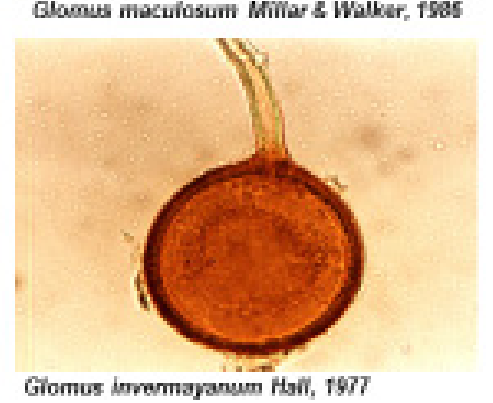

Giomes imremayanum $\mathrm{HaN}, 1977$

Figura 6. Diversidad de morfoespecie de HMA aisladas de suelos compactados y no compactados pertenecientes al municipio de San Marcos, departamento de Sucre, Colombia. Año 2013. Fuente: Espitia-Doria, 2013. 


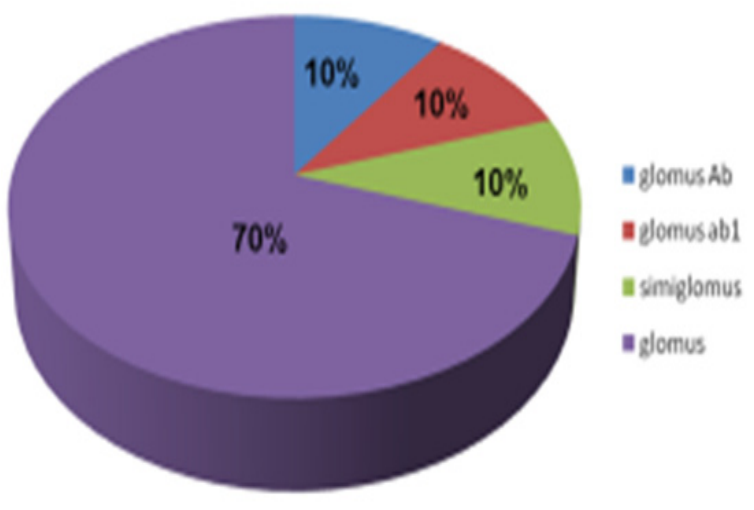

Figura 7. Porcentaje de Géneros de HMA, aislados en suelos del municipio de Sincelejo, departamento de Sucre, Colombia. Año 2013.

En estudios de suelos en la Amazonia Colombiana, PEÑA-VENEGAS et al., (2007) encontró 18 morfotipos de esporas de HMA diferentes, 11 especies de Glomus, 4 especies de Acaulospora, y una especie de Archaeospora, Scutellospora y Gigaspora. Diversos autores han mostrado como los géneros más representativos de suelos tropicales húmedos de textura arcillosa a franco-arcillosa son Glomus y Acaulospora, siendo los géneros Gigaspora y Scutellospora los menos frecuentes (BHATIA et al., 1996). Esta estructura en la composición micorrízica de los suelos ha sido igualmente reportada para la región amazónica venezolana (CÁCERES, 1989), y brasileña (CAPRONI et al., 2003). De los tres trabajos anteriormente citados, se encuentra que el género Glomus representa más del $50 \%$ de la diversidad micorrízica del suelo, corroborando los resultados de este estudio.

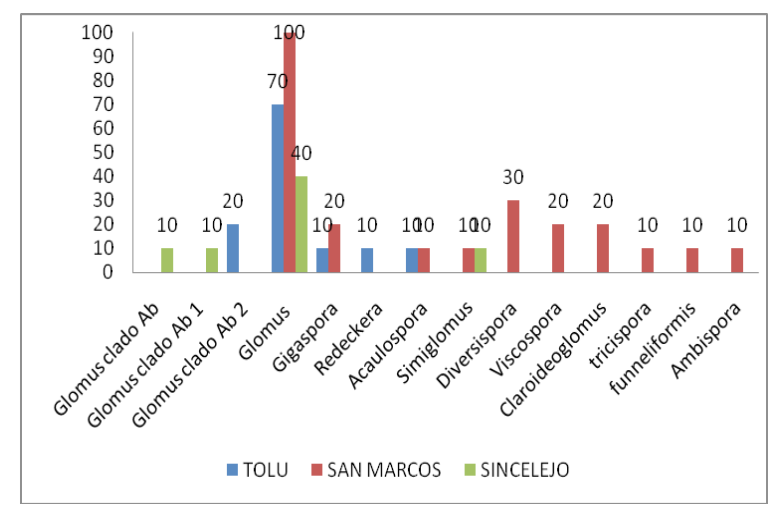

Figura 8. Porcentaje de géneros de HMA aislados en agroecosistemas de suelos sembrados con pasto colosoana en tres municipios del departamento de Sucre, Colombia. Año 2013.

\section{Conclusiones}

Las mayores densidades de esporas fueron observadas en el municipio de Tolú para suelos compactados con respecto a Sincelejo y San Marcos. Asimismo, se observó mayores valores de densidad de HMA en suelos no compactados para este mismo municipio con respecto a los otros analizados. En suelos no compactados, ocurre esporulación de HMA cuando en los suelos se encuentra valores altos de fósforo, sodio y calcio.

Un total 50 morfoespecies fueron aislados de suelos nativos y compactados sembrados con pasto colosoana. El género de HMA que más predomina en los suelos compactados y nativos de los tres municipios seleccionados fue el género Glomus, seguido de otros géneros en menor proporción. Los suelos que presentaron mayor riqueza de géneros de HMA fue el municipio de San Marcos, con respecto a los suelos del municipio de Sincelejo, quienes mostraron la menor riqueza de morfoespecies de HMA.

Alrededor del $50 \%$ del territorio del municipio de San Marcos se destina a actividades agropecuarias; la representación de ecosistemas de humedal (integrado por las categorías de: suelos anegados, ciénagas, complejo humedal ciénaga, complejo arbustivo humedal y humedal) cubre el $30 \%$ del área municipal (PLAN DE GESTIÓN AMBIENTAL REGIONAL-CORPOMOJANA, 2012).

Las zonas agrícolas, en el municipio de San Marcos corresponde a los suelos de valle y planicie, en clima cálido húmedo, relieves planos o inclinados, pendientes no mayores del $7 \%$ y altitudes que no sobrepasan los 100 m.s.n.m. los suelos se desarrollan a partir de depósitos aluviales, caracterizados por tener contenidos de gravilla en el perfil y, en algunas pequeñas áreas, piedras en la superficie; hay limitaciones por fluctuaciones del nivel freático. Las zonas ganaderas, las unidades apropiadas para esta actividad corresponden al lomerío. Esta zona se caracteriza por ser de suelos arcillosos, ácidos, con abundante gravilla y cascajo; relieve plano a ondulado profundidad efectiva, con fragmentos rocosos en superficie y a través del perfil, su morfo dinámica es de tipo mixto erosionable con grado leve a moderado (PLAN BÁSICO DE ORDENAMIENTO TERRITORIAL, 2008).

Agradecimientos: Los autores agradecen a colaboración brindada por los estudiantes del programa de Biología de la Universidad de Sucre, Roberto Carlos Jiménez Zappa y Ader Alfredo Núñez Ortega. 


\section{Referencias}

AGUILERA, M.D. 2005. Economía Regional: La Economía del Departamento de Sucre: Ganadería y Sector Público. Editorial Banco de la República. Colombia.

BARRIOS, E. 2007. Soil biota, ecosystem services and land productivity. Ecological economics 64:269-285.

BHATIA, N. P.; SUNDARI, K.; ADHOLEYA, A. 1996. Concepts in Mycorrhizal Research: Diversity and selective dominance of vesicular-arbuscular mycorrhizal fungi. Handbook of Vegetation Science. Editorial Kluwer Academic Publishers, USA..

BENDING, G.; TURNERA, M.; RAYNSB, F.; MARXCAND, M.; WOOD, M. 2004. Microbial and biochemical soil quality indicators and their potential for differentiating areas under contrasting agricultural management regimes. Soil Biology and Biochemistry 36 (11):1785-1792.

BOTERO, M. 1998. Influencia de las micorrizas arbusculares sobre el crecimiento del Aliso (Almus acuminata H.B.K. Subsp acuminata) en tres suelos diferentes de Colombia. Santafé de Bogotá, Tesis (Maestría en Microbiología). Universidad de los Andes. Facultad de Ciencias. Departamento de Ciencias Biológicas. 90 p.

CÁCERES, A. 1989. Las micorrizas vesiculo-arbusculares en un bosque húmedo tropical y su evolución luego de la perturbación (conuco) y la sucesión por 60 años en San Carlos de Río Negro. (Tesis de Maestría). Centro de Estudios Avanzados, Instituto Venezolano de Investigaciones Científicas IVIC, Caracas, Venezuela.

CAPRONI, A.L.; FRANCO, A.A.; BERBARA, R.L.L.; TRUFERRI, S.B.; DE OLIVEIRA, J.R.D.; MONTEIRO, A.B. 2003. Ocorrência de fungos micorrizicos arbusculares en áreas revegetadas após mineração de bauxita en Porto Trombetas, Pará. Pesq. Agropec. Bras. Brasilia. 38(12): 1409-1418.

CHAMORRO, D. 1994. Informe final de investigaciones en forrajes tropicales. Editorial CORPOICA. Grupo Regional Pecuário. Santiago de Cali, Colombia.

DEPARTAMENTO ADMINISTRATIVO NACIONAL DE ENCUESTA. 1996. Encuesta Nacional Agropecuaria, Resultados 1995, Costa Atlántico. Bogotá, Colombia.

DANIELS, B.; SKIPPER, H. 1991. Methods for the recovery and quantitative estimation of propaqules from soil. Editorial APS Press. The American Phitopathological Society St. Paul. USA.

ESPITIA, F.; MARTíNEZ, E. 2003. Identificación de Hongos Formadores de Micorrizas Vesículo Arbusculares asociados al pasto colosoana y angletón en los Municipios de Corozal y Tolú. (Trabajo de Grado). Facultad de Educación y Ciencias, Universidad de Sucre.

GONZÁLEZ, A. 1996. Las micorrizas como biofertilizantes en la agricultura. Curso cultivo e investigación del chontaduro. Editorial CORPOICA. Colombia.

GOTO, B.T.; MAIA, L.C.; OEHL, F. 2008. Ambispora brasiliensis, a new ornamented species in thearbuscular mycorrhizaforming Glomeromycetes. Mycotaxon 105:11-18.

INTERNATIONAL COLLECTION OF ARBUSCULAR AND VESICULARARBUSCULAR MYCORRHIZAL- INVAM. 2002. Web http://invam.caf.wvu.edu/. Noviembre/10/2002.

INTERNATIONAL CULTURE COLLECTION OF VESICULAR ARBUSCULAR MYCORRHIZAL FUNGI-INVAM. 2013. General life cycle and the structures formed. Web http://invam.caf.wvu.edu/. Julio/13/2013.

KASCHUK. G.; ALBERTON, O.; HUNGRIA, M. 2010. Three decades of soil microbial biomass studies in Brazilian ecosystems: Lessons learned about soil quality and indications for improving sustainability. Soil Biology and Biochemistry 42(1): 1-13. 
MECINAS, L.J.; DOOR, R.C.; CHUNG, M.A.; MORENO, D.P. 1991. Micorrizas en tres especies forestales de la Amazonia peruana. Rev For Peru 18:29-43.

MORTON, E. 1996. Classification and identification of mycorrhizal fungi- INVAM, Firs ICOM Workhosp. Wirginia, USA.

OEHL, F.; DA SILVA, G.; TOMIO, B.; SIEVERDING, E. 2011. Glomeromycota: three new genera and glomoid species reorganized. Mycotaxon 116:75-120.

OEHL, F.; SIEVERDING, E.; PALENZUELA, J.; INEICHEN, K.; SILVA, GA. 2011. Advances in Glomeromycota taxonomy and classification. IMA Fungus 2(2) 191-199.

PEÑA-VENEGAS, C.; CARDONA, G.; ARGUELLES, J.; ARCOS, A. 2007. Micorrizas arbusculares del sur de la amazonia colombiana y su relación con algunos factores fisicoquímicos y biológicos del suelo. Acta Amazónica. 37 (3):327-336.

PEÑA, C.; CARDONA, G.; MAZORRA, A.; ARGUELLEZ, J.; ARCOS, A. 2006. Micorrizas arbusculares de la Amazonia Colombiana. Catálogo ilustrado. Instituto amazónico de investigaciones científicas - SINCHI. Leticia, Amazonas, Colombia.

PEREZ, C.R.; VERTEL, M. M.; PEREZ, C. A. 2015. Efecto de diferentes tipos de abonos sobre hongos edáficos en el agroecosistema de Bothriochloa pertusa, (L) A. Camus, en Sabanas sucreñas, Colombia. Livestock Research For Rural Development $27(1): 1-10$.

PICONE, C. 2000. Diversity and abundance of arbuscular-mycorrhizal fungus spores in tropical forest and pasture. Biotropica 32 (4a):734-750.

PLAN BÁSICO DE ORDENAMIENTO TERRITORIAL. 2008. Diagnóstico biofísico del municipio de San Marcos departamento de Sucre. Editorial Alcaldía de San Marcos. Colombia.

PLAN DE GESTIÓN AMBIENTAL REGIONAL-CORPOMOJANA, 2002-2012. 2013. Fase I. Fondo de Compensación Ambiental - Asociación de Corporaciones. Departamento de Sucre. Sincelejo, Colombia.

SÁNCHEZ, M. 1999. Endomicorrizas en agroecosistemas Colombianos. Editorial Universidad Nacional de Colombia, sede Palmira. Colombia.

SANT'ANNA, S.; FERNANDES, M.; IVO, W.; COSTA, J. 2009. Evaluation of Soil Quality Indicators in Sugarcane Management in Sandy Loam Soil. Pedosphere 19 (3):312-322.

SCHENCK, N.; PEREZ, Y. 1990. Manual for the identification of VA mycorrhizal fungi. Tercera Edición. USA: Synergistic Publications. USA.

SCHENCK, N.C.; SIQUEIRA, J.O.; OLIVEIRA, E. 1989. Changes in the incidence of VA mycorrhizal fungi with changes in ecosystems. Developments in Soil Science (18):125-129.

SCHLOTER, M.; DILLY, O.; MUNCH, J.C. 2003. Indicators for evaluating soil quality. Agriculture, Ecosystems \& Environment 98 (1):255-262.

SIEVERDING, E. 1983. Manual de métodos para la investigación de las micorrizas arbusculares en el laboratorio. Centro Internacional de Agricultura Tropical, CIAT. Colombia.

SIEVERDING, E.; OEHL, F. 2006. Revision of Entrophospora and description of kuklospora and intraspora, two new genera in the arbuscular mycorrhizal Glomeromycetes. Journal of Applied Botany and Food Quality 80:69-81.

UNIVERSIDAD DE SUCRE. 1996. Situación actual del sectorganadero en el departamento de Sucre. Sincelejo, Colombia.

WINDINGA, A.; HUND-RINKEB, K.; RUTGERS, M. 2004. The use of microorganisms in ecological soil classification and assessment concepts. Ecotoxicology and environmental 62 (2):230.248. 